\title{
Breakthrough Treatment of "Dengue Viral Infection" The Report on the Dengue Viral Epidemic of 2011 in Province of Punjab Pakistan.
}

\author{
Prof. Dr. Shahid Hussain Sheikh, \\ M.B.B.S. (Pk), Ph.D. (SL), FACPE (USA), M.I.S.I.D. (USA). Chairman: NIDS Treatment \& Research Center \\ Department of Neurology \& Neuro Virology United Christian Hospital, Gulberg III, Lahore, Pakistan
}

\begin{abstract}
The present paper aimed to overcome the Viral Pathology in the emerging Dengue Virus Epidemic. Since 2010 it is a second epidemic of dengue Fever (DF) and dengue Hemorrhagic fever (DHF) that has claimed over 300 lives in the Punjab area. There has been no set treatment protocol other than the World Health Organization (WHO) guidelines to treat this viral infection. Many physicians tried to follow the same and achieved some degree of success but unfortunately mismanagement of Dengue inflicted patients led to a high mortality. Malaysian physicians reported to have modified the WHO protocol to fit the needs of the local epidemic in 2009. However; the mainstay of the treatment was the balance of fluid dynamics of the patient's body. Unfortunately, the depleting platelets counts have been a media promoted scare of the dengue fever and dengue hemorrhage fever. The depleting Platelets count is not the only indication of the DF and DHF. However, its drastic reduction below 150,000 is the leading cause of bleeding (Internal \& External) and eventual death. To date, the Pathophysiology that causes the symptoms of DF \& DHF and the neurological deficits have been very poorly understood. Therefore, the treatment protocol presented by the WHO and the local medical practioners has been deemed partial or insufficient. Great number of patients has been seen with the recurrence of the dengue fever or incomplete treatment or the extensive dengue infection leading to bleeding, neurological deficit or directly comatose and death. Ever changing patient admission guidelines also led to the high risk of patient mortality. Therefore, the treatment protocol designed by us has successfully addressed the DF \& DHF challenged patient population most effectively, resulting into no platelets transfusion and zero patient mortality. This unique project entertained 1298 patients from the greater community of city of Lahore. Majority of patients were seen as a direct visit to the UCH Dengue clinic, and many were received as a referral from other hospitals.
\end{abstract}

Keywords: Dengue Virus, Dengue Fever.

\section{Introduction}

Most people infected with any of the four forms of dengue virus will not develop any symptoms or at most will have a mild fever. However, 50 to 100 million people a year do suffer the fever, headache, and severe joint pain that give the disease its nickname of break-bone fever. Sadly, some 20,000 people-mostly children and young adults experiencing their second episode of infection — die of dengue annually [1].

The severe forms of dengue infection occurs when blood plasma leaks out of blood vessels into the surrounding tissues, which can lead to a drop in the blood pressure, state of shock prevails and, if left untreated, death results.

Central \& peripheral Nervous System is the most vulnerable to the Dengue Viral RNA proliferation in conjunction with the Herpes Viral RNA compromising the Neuronal and Nerve homeostasis.

Since 2010 it is a second epidemic of dengue fever (DF) and dengue Hemorrhagic fever (DHF) that has claimed over 300 lives in the Punjab area. There has been no set treatment protocol other than the World Health Organization (WHO) to treat this virus. Many physicians tried to follow the same and achieved some degree of success, unfortunately mismanagement of Dengue inflicted patients led to a high mortality.

To date, the neurological Pathophysiology that cause the symptoms of DF \& DHF have been very poorly understood.

In the most healthcare institutions the priority has been shifted to fluid dynamics rather than the neurological deficits.

The neuro invasivity of the dengue virus has been grossly overlooked, that has been presented as the prime symptom from the very beginning. All patients presented the following Symptomatology.
1. Fever ranging from $99 \circ \mathrm{F}$ to $106 \circ \mathrm{F}$
2. Vomiting / Belly Aches
3. Extensively Lethargic
4. Mild to Severe Headaches
5. Pain behind eyeballs
7. Lower Legs weakness
6. Extensive Weakness
8. Nose / Gums Bleeding 
9. Extensive Menstrual Bleeding

11. Severe Headaches.

13. Low count of Platelets.
10. Confused / Disoriented

12. Exaggeration of chronic illness

Accordingly the WHO recommended treatment strategy has left a major morbidity factor unaddressed.

Therefore, the treatment protocol presented by the WHO and the local medical practioners has been deemed partial or insufficient. Great number of patients has been seen with the recurrence of the dengue fever or incomplete treatment or the extensive dengue infection leading to bleeding, neurological deficits or directly comatose and death [2].

The treatment protocol we designed, addressed to begin with the internal bleeding and neurological deficits caused by the Dengue Viral RNAs. This successfully resulted into no plasma leak no platelets transfusion and zero patient mortality.

This unique project entertained over 1298 patients from the greater community of city of Lahore. Majority of patients were seen as a direct visit to the UCH Dengue clinic, and many received as a transfer from other hospitals [3].

\section{Materials \& Methods}

From September 24, 2011 to November 30, 2011, 1298 patients were registered in our Dengue Fever program.

Patients ranging from age 9 years to 68 years old, males \& females. From this pool of patients 300 patients were admitted with Platelets profile ranging from 0 to 100,000 and treated for the dengue fever and dengue hemorrhagic fever. impairments.

All patients presented some degree of neurological deficits from headaches to serious neurological

Strategy to first address the Neurological disorder was a highly appropriate and vitally needed to save the lives. In this investigation we designed the following Dengue Patient Care Treatment Protocol and administered to over 300 in-patients that resulted into zero mortality.

The patients were categorized into 3 Units.
A. High Dependency Unit
B. Intermediary Unit
C. General Unit

The following strategic prescription was administered to counter the neurological deficits.

1. Ringer Lactate 500 - $1000 \mathrm{ml}$ (Non-Diabetics) / N/S 0.9\% 500ml (Diabetics).

2. Prevalent vitamins $1 \mathrm{ml}$ (I.V.) B.I.D. 3 to 4 days.

3. Plasma expander $500 \mathrm{ml}+$ Injectable multivitamin O.D. (I.V.) 3-5 days (If the Platelets are $<100,000$ )

4. Antipyretic $500 \mathrm{mg}$ T.I.D. until the fever is down to normal. (Tabs)

5. Coagulant $500 \mathrm{mg}$ (I.V) Stat / s.o.s (If the Platelets are < 100,000) and bleeding patients.

6. PPI (I.V.)

7. Monitoring patient's vital every 4 to 6 hours.

8. Monitor Blood Profile daily $(\mathrm{CBC}) /$ twice a day for serious patients.

9. Discharge patients when the platelets were at 150,000 levels and fully recovered.

Drastically falling Platelet count has scared the masses inflicted with the DF \& DHF. Many hospitals opted for the transfusion of the platelets. No patient was given any Platelet transfusion and/or blood transfusion.

The reproduction of the platelets of our patients was successfully stimulated by the infusion of plasma expander with the combination of multivitamins.

This resulted in the production of 10,000 to 30,000 platelets in the first 2 days and then almost doubling the 2 nd day quantity on the 3 rd to 5 th day. Fever on the other hand was managed in the first 2 days by the administration of Antipyretics.

\section{Results}

After a successful recovery with average of 5 days of hospitalization, 300 patients have been discharged fully healthy, from the hospital. 


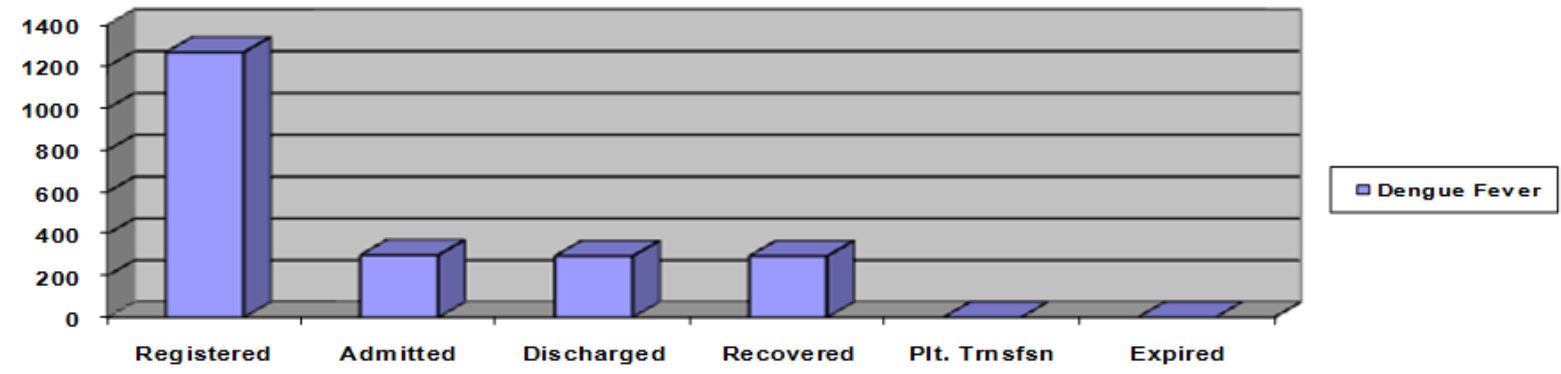

The above medicines were administered as a standard treatment protocol indiscriminately to all dengue inflicted Patients. Each patient revealed a definitive progress in the Platelets production by 10,000 to 30,000 on the average in first 2 days. Whereas, a double production of the platelets seen on the 3rd to 5th day until fully recovered.

Internal and external bleeding was stopped by coagulant therapy given directly via I.V. and monitored vigilantly for further bleeding; platelets profile and blood pressure.

Dengue fever was seen resolved as the production of platelets improved. Average hospitals stay of the patient recorded was 5 days. All neurological disorders were seen resolved in the first 24 hours. Every patient was discharged as soon as full recovery was realized.

Due to the ventilator dependency, one patient referred from sheikhupura was redirected to the local government hospital. No patient expired.

\section{Discussion}

At the tertiary care center in India, a clinical observation was made by the Medical Scientists on 26 patients inflicted by the Dengue fever almost at the same time when we were treating the patients at the UCH, Lahore. They found and reported the neurological deficits consistent with the diagnosis of the following:

1. Guillain Barre Syndrome

2. Hhypokalemic paralysis

3. Viral myositis

4. Opsoclonus-myoclonus syndrome

5. Myelitis

6. Acute disseminated encephalomyelitis

7. Brachial neuritis and Opsoclonus-myoclonus syndrome were observed for the first time. [2]

Dengue Virus is the RNA that thrives in the anaerobic environment and its protein compliments the existing other viral RNA proliferation i.e. Herpes family of viral RNA's in the nucleus of the pre-infected neurons of the Brain [4].

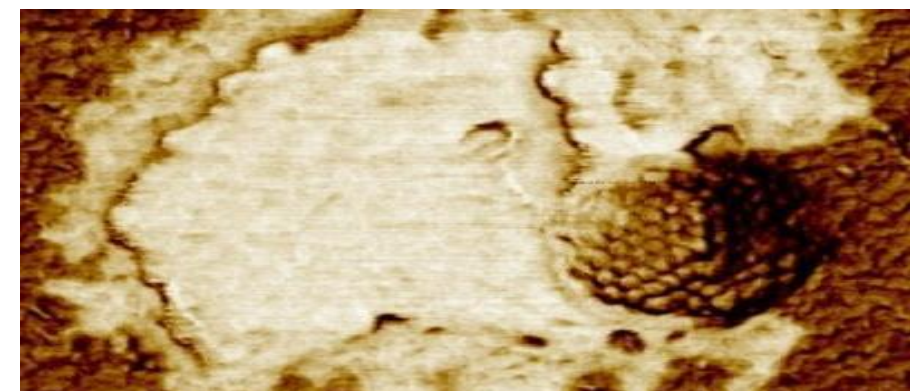

Neuronal Lesion due Herpes virus

Atomic Force Microscopic Real Time Image of Neuronal Infection

Permitted by: Marco Plomp, Alexander McPherson, and Alexander J. Malkin

Department of Molecular Biology and Biochemistry, University of California, Irvine, California [4].

Neurological deficits encountered due to the dengue viral infection are possibly rationalized as following:

1. Herpes Viral RNA proliferation already exists among pre-exposed patients. Sub Nucleus RNA proliferation offsets the neuronal homeostasis. Low level neuronal cytopathy promotes as amino acid intake empowers the HSV RNA proliferation. [5]

2. Dengue Viral RNA is the most simplified Amino Acid strengthened by the fibrinogen of platelets, either complimenting the HSV or directly attacking the neuronal mitochondria or sub nucleus RNA to disrupt homeostasis. 
3. Prevalence of the autoimmune disorder to increase the neuron's nucleus dysfunction.

Fact remains that multifocal neuronal homeostasis is compromised; therefore, neurological disorders prevail right from the onset of the Dengue Fever. The chronological order seen was as follows as is consistent and aligned with the observation made by the Indian Scientist.

1. Fever ranging from $99 \circ \mathrm{F}$ to $106^{\circ} \mathrm{F}$

3. Extensively Lethargic

4. Mild to Severe Headaches

6. Extensive Weakness

7. Lower Legs weakness

9. Extensive Menstrual Bleeding

10. Confused / Disoriented

12. Exaggeration of chronic illness
2. Vomiting / Belly Aches

5. Pain behind eyeballs

8. Nose / Gums Bleeding

11. Severe Headaches.

13. Low count of Platelets.

Further research needs to focus on the viral RNA proliferation against the Immunity and Dengue viral impact on the sick neurons. Our strategy focused to rescue the neurons from both of the above scenarios.

Therefore, the use of vitamin $\mathrm{B}_{12}$ helped us break the above scenarios by stimulating the Hb production and compensating for the lost Platelets and readily absorbing in the neurons to recover and maintain the Neurological homeostasis.

Parallel Coagulation therapy helped us avoid the intra cellular plasma leakage, so that, the drop in the pulse pressure could be refrained. Patients recovered from this viral epidemic on an average of 5 days.

\section{Conclusion}

Dengue Fever management program was a very successful endeavor at the UCH with the collaboration of PMA and the Government of Punjab. The treatment protocol designed by us addressed the most vital urgency of defending against the "Neurological Deficits" and to save the lives. Therefore, the nagging pains, general weakness and bleeding was the top of our treatment agenda. The fluid dynamics has played a significant role in the patient recovery. This resulted in zero mortality.

\section{Special thanks to:}

\section{Acknowledgements}

A. Dr. Emmanuel Bhatti the Medical Director of the United Christian Hospital, Lahore for clinical support and critical review of the study and the paper.

B. All the doctors of the Pakistan Medical Association, in particular Dr. Adnan Sheikh., Dr. Amna Ejaz, Dr. Ejaz Malik, Dr. A. Sherwani, Dr. Ashraf Nizami, Dr. Izhar Chaudhry, Dr. Tanvir Islam, Dr. Mujahid Hussain for their support in the Clinical follow up.

C. Mr. Francis Sodagar, Mr. Jamshed Irfan, Ms. Stella for their Laboratory Support.

[1]. Dengue Report, World Health Organization, 2010.

\section{References}

[2]. Garg RK, V Atam, R V erma, P S harma, MK Singh and HS Mehrotra, 2011.Neurological complications of dengue fever: Experience from a tertiary center of north India. Ann Indian Acad Neurol. 2011 Oct;14(4):272-8 http://www.ncbi.nlm.nih.gov/pubmed/22346016/

[3]. Gov't of Punjab, Department of Health, Dengue Viral Epidemic 2011.

[4]. Plomp M, A McPherson and A. Malkin, 2002. Atomic Force Microscopy. AJP June 2002, Vol. 160, No. 6

[5]. Sheikh S. , 2012.Breakthrough Treatment of Hypertension, IOSR-JDMS: 2012 Sep-Oct, Vol 1, No. 5:33-38 